\title{
Application of 3.0T magnetic resonance arterial spin labeling (ASL) technology in mild and moderate intracranial atherosclerotic stenosis
}

\author{
ZHONGWEI LI $^{1 *}$, NAIKUN LI ${ }^{2 *}$, YANYAN QU ${ }^{3}$, FENG GAI $^{4}$, GUOWEI ZHANG ${ }^{1}$ and GUANGHUI ZHANG ${ }^{2}$ \\ ${ }^{1}$ CT Room, Yantaishan Hospital, Yantai, Shandong 264001; ${ }^{2}$ Department of Medical Imaging, \\ Yantai Affiliated Hospital of Binzhou Medical University, Yantai, Shandong 264100; ${ }^{3}$ Department \\ of Endocrinology, Yantaishan Hospital, Yantai, Shandong 264001; ${ }^{4}$ Department of Radiology, \\ Yantai Municipal Laiyang Central Hospital, Yantai, Shandong 265200, P.R. China
}

Received March 3, 2015; Accepted May 6, 2016

DOI: $10.3892 /$ etm.2016.3318

\begin{abstract}
The application value of 3.0T magnetic resonance arterial spin labeling (ASL) technology in mild and moderate intracranial atherosclerotic stenosis was evaluated. A total of 58 cases of transient ischemic attack (TIA) and 60 cases of ischemic cerebral apoplexy cases were selected. The cases were analysed using a GE Healthcare Signa HDx 3.0T superconducting whole-body magnetic resonance scan within $24 \mathrm{~h}$ of attack. Eight-channel head phased array coils and conventional sequence were used to create T1-weighted images (T1WI), T2WI, diffusion-weighted imaging, magnetic resonance angiography (MRA) and ASL imaging, which were generated into ASL pseudo-color images (blue was hypoperfusion area) through post-processing in order to compare and analyze the correlation and differences between ASL and conventional imaging in terms of lesion location, size, blood perfusion situation and signal range of relative cerebral blood flow (rCBF). The results showed that, 13 TIA cases of abnormal signal in conventional magnetic resonance imaging (MRI) can also be found through ASL technology. Diameter stenosis beyond 30\% in MRA can also be tested in ASL. A positive rate in ASL was significantly higher than that of conventional MRI $\left(\chi^{2}=29.078, P<0.001\right)$ and hypoperfusion area was greatly increased $(\mathrm{t}=32.526, \mathrm{P}<0.001)$. The $\mathrm{rCBF}$ value was positively correlated with the degree of diameter stenosis shown in MRA ( $\mathrm{r}=0.524, \mathrm{P}=0.012)$. Additionally, the positive rate of ASL was positively correlated with the attack times of TIA
\end{abstract}

Correspondence to: Dr Feng Gai, Department of Radiology, Yantai Municipal Laiyang Central Hospital, 121 Changshan Road, Yantai, Shandong 265200, P.R. China

E-mail: 1i_zhongwei1@163.com

*Contributed equally

Key words: magnetic resonance arterial spin labeling technology, transient ischemic attack, ischemic cerebral apoplexy $(\mathrm{r}=0.352, \mathrm{P}=0.027)$. A total of 39 cerebral apoplexy cases of abnormal signal in conventional MRI were also found through ASL technology. A positive rate in ASL was significantly higher than that of conventional MRI $\left(\chi^{2}=7.685, \mathrm{P}=0.006\right)$ and hypoperfusion area was greatly increased $(\mathrm{t}=9.425, \mathrm{P}<0.001)$. The $\mathrm{rCBF}$ value was positively correlated with the degree of diameter stenosis $(\mathrm{r}=0.635, \mathrm{P}=0.009)$. In conclusion, 3.0T ASL correlated with early diagnosis of TIA and mild and moderate intracranial arterial stenosis of cerebral apoplexy.

\section{Introduction}

Ischemic cerebral apoplexy is a worldwide. It is reported that the prevalence of ischemic stroke is $20-40 \%$ with a high disability rate $(60-85 \%)$ and high mortality rate $(30-45 \%)$ for individuals over the age of 40 years (1) It is believed that multiple factors result in the temporary or permanent ischemia of the artery. Furthermore, there are a series of secondary mechanisms of brain tissue and cells e.g., ischemic/hypoxic injury, energy metabolic disorder, oxidative stress and calcium overload (2). Approximately 50-65\% patients have transient ischemic attack (TIA), which may be a different development process for another disease compared with cerebral apoplexy, but they have the same pathological basis i.e., stenosis of feeding artery (3). Early diagnosis of the target vessel lesion was significant in terms of improving the clinical remedy rate and life quality. Conventional magnetic resonance imaging $(\mathrm{MRI})$ has a resolution ratio on components of brain tissue, but also has poor results on showing blood flow perfusion (4). Dynamic magnetic sensitivity contrast agent perfusion-weighted imaging (PWI) increases complications and counterfeit image interference from exogenous contrast agents (5). Magnetic resonance angiography (MRA) has unsatisfactory development on small artery lesions, small and moderate artery with mild or no obvious stenosis (6). It cannot provide accurate imaging information in time. Cerebral blood flow $(\mathrm{CBF})$ imaging, which is obtained through using free water proton in arterial blood as an endogenous tracer and reverse pulse technology for labeling and then post-processing, is able to sensitively and specifically indicate abnormal changes of 
cerebral perfusion (7). It has been approved to different degrees for ischemic and hemorrhagic cerebrovascular diseases, and has better developing effects compared with conventional diffusion-weighted imaging (DWI), PWI and functional MRI, and also better signal-to-noise ratio (SNR), high repeatability and consensus stability (8).

The present study examined the application value of arterial spin labeling (ASL) in TIA and mild and moderate intracranial atherosclerotic stenosis, which provide references for the early recognition of target lesions and immediate treatment clinically.

\section{Patients and methods}

Patients. A total of 58 cases of TIA and 60 cases of ischemic cerebral apoplexy were diagnosed at the Yantaishan Hospital (Shandong, China) and were selected between January 2014 and January 2016. These cases were 32 men and 26 women in the TIA group, with an average age of $45.6 \pm 10.3$ years. The average onset time of disease was $1.5 \pm 0.4 \mathrm{~min}$, and the average number of attack times was $1.5 \pm 0.6$. There were 12 hypertensive, 8 smoking, 5 diabetic and 7 hyperlipidaemic cases. In the cerebral apoplexy group, there were 36 male and 34 female cases, with an average age of $47.7 \pm 11.5$ years, an average onset time of disease of $26.4 \pm 7.2 \mathrm{~min}$, and an average number of attack times of $1.3 \pm 0.5$. There were 32 cases with lesion location at basal ganglia region, 14 cases at cerebrum, 7 cases at cerebellum and 7 cases at brainstem.. There were 15 hypertensive, 10 smoking, 3 diabetic and 5 hyperlipidaemic cases. There was no statistical differences between the two groups regarding gender, age, attack times and combined disease type $(\mathrm{P}>0.05)$.

Infored consent was obtained from the patients and their family members. Approval for the study was obtained by the ethics committee of Yantaishan Hospital.

Study method. GE Healthcare Signa HDx 3.0T (GE Healthcare, Piscataway, NJ, USA) superconducting whole-body magnetic resonance scan was performed on all the patients within $24 \mathrm{~h}$ of attack. The maximum uniaxial gradient strength was $\geq 45 \mathrm{mT} / \mathrm{m}$. Eight-channel head phased array coils and conventional sequence were used to obtain T1-weighted images (T1WI), T2WI, DWI, MRA and ASL imaging. The scanning parameters were OAxT1WI: TR=1,750 msec, TE=24 msec, number of layers was 20, thickness of layer was $5.0 \mathrm{~mm}$, spacing between layers was $1.0 \mathrm{~mm}$, vision FOV: 22x22 cm, NEX was 1 and matrix was 320x224. OAxT2WI: TR=5,100 $\mathrm{msec}$, $\mathrm{TE}=118.9 \mathrm{msec}$, number of layers was 20 , thickness of layer was $5.0 \mathrm{~mm}$, spacing between layers was $1.0 \mathrm{~mm}$, FOV: 22x22 cm, NEX was 1.5 and matrix was $512 \times 512$. DWI: TR=5,400 msec, $\mathrm{TE}=$ minimum, number of layers were 20 , thickness of layer was $5.0 \mathrm{~mm}$, spacing between layers was $1.0 \mathrm{~mm}$, vision FOV: $22 \times 22 \mathrm{~cm}$ and NEX was 1 . MRA selected three-dimensional time-of-flight method and $\mathrm{TR}=40 \mathrm{msec}, \mathrm{TE}=8 \mathrm{msec}$, number of layers was 20 , thickness of layer was $5.0 \mathrm{~mm}$, spacing between layers was $1.0 \mathrm{~mm}$, vision FOV: $22 \times 22 \mathrm{~cm}$ and NEX was 1 . Intracranial vascular examination included intracranial artery (intracalvarium, siphon bend and terminal segment), middle cerebral artery M1-3 segments and anterior cerebral artery A1-3 segments.
For ASL technology, FAIR was used and TR=1,000 msec, $\mathrm{TE}=13.8 \mathrm{msec}, \mathrm{TI}=2,000 \mathrm{msec}$, number of layers was 20 , thickness of layer was $1.3 \mathrm{~mm}$, spacing between layers was $1.0 \mathrm{~mm}$, vision FOV: 22x22 cm, NEX was 80, matrix was 256x128 and scanning time was $2 \mathrm{~min}$ and $48 \mathrm{sec}$. Scanning was performed at the plane of $1 \mathrm{~cm}$ above the corpus callosum, because this area was supplied with blood from the anterior cerebral and middle cerebral arteries, and therefore was less influenced by magnetic sensitivity or vein pollution. Reconstruction of ASL imaging was shown in pseudo color map, where the red area was high perfusion, green was moderate perfusion and blue was low perfusion. A qualitative and semiquantitative analysis on ASL imaging was performed and the corresponding value of relative $\mathrm{CBF}$ ( $\mathrm{rCBF}$ ) was used to distinguish perfusion, where $\mathrm{rCBF}<0.9$ referred to low perfusion, 0.9-1.1 was moderate perfusion and $>1.1$ high perfusion. Two experienced MRI doctors jointly observed the distribution of grey and white matter, and subsequently confirmed the abnormal area of perfusion and determined the lesion location based on clinical manifestations of TIA. The doctors manually drew the region of interest (ROI) for grey and white matter of abnormal perfusion. RESTplus 1.1 software (RESTplus, Hangzhou, China; http://restfmri.net/forum/index.php?q=rest) was used to manually test ROI was used to manually test ROI signal strength, and the image method was used to place ROI at the other side to measure signal strength of the corresponding side and perform semiquantitative analysis. The average signal strength at the abnormal perfusion side was the healthy side, and was considered abnormal if the value was $>20 \%$.

Observation index. The correlation and differences between ASL and conventional imaging were compared and analyzed in terms of lesion location, size, blood perfusion and the signal range of $\mathrm{rCBF}$.

Statistical analysis. SPSS 19.0 (SPSS, Inc., Chicago, IL, USA) was used to analyze and process data. Quantitative data were presented as mean value \pm standard deviation, where comparisons between groups were made using a t-test. Qualitative data were presented as number of cases, or percentage, and comparisons between groups were made using the $\chi^{2}$ test. Correlation analysis was performed using Pearson's or Spearman's tests. $\mathrm{P}<0.05$ was considered to indicate a statistically significant difference.

\section{Results}

Analysis on the development in TIA group. There were 13 cases of an abnormal signal tested in conventional MRI (manifesting as T1 and T2 low signal) and the positive rate was $22.4 \%$. The hypoperfusion area of DWI was $0.5-1.8 \mathrm{~mm}^{2}$ and the average was $1.1 \pm 0.6 \mathrm{~mm}^{2}$. The diameter stenosis of intracranial arterial lumen tested in MRA was 10-50\% and average was $32.5 \pm 10.4 \%$. In ASL, 37 cases with hypoperfusion area were tested (63.8\%), the rCBF was 0.6-1.2 and the average was $0.8 \pm 0.3$. The semiquantitative analysis revealed that the rate of abnormal perfusion signals was $72.4 \%(42 / 58)$, the hypoperfusion area was $1.2-5.4 \mathrm{~mm}^{2}$ and the average was $3.3 \pm 1.4 \mathrm{~mm}^{2}$. For the location of the hypoperfusion area, 18 cases were at basal ganglia, 10 cases were at cerebrum, 

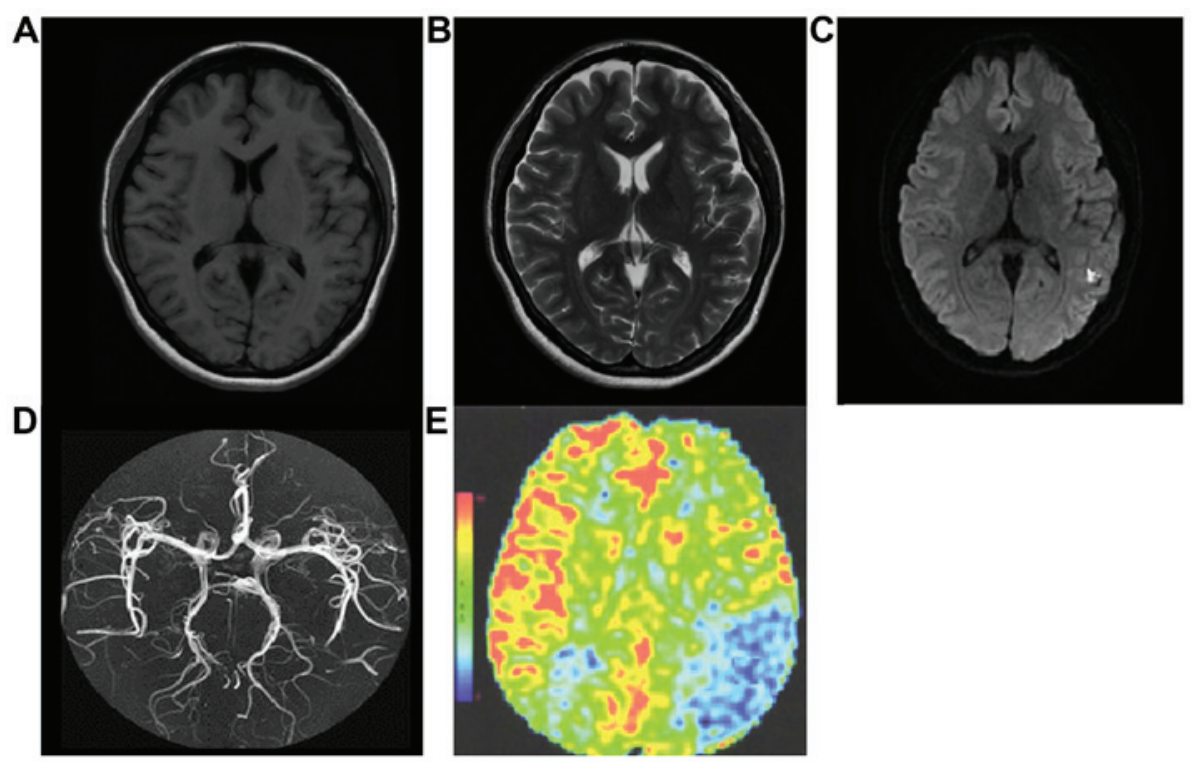

Figure 1. TIA scan (A) T1. (B) T2 with no obvious changes. (C) Small ischemic foci at left temporal-occipital junction of DW1. (D) MRA with no obvious arterial luminal stenosis. (E) Hyperfusion area of left temporo-occipital junction of ASL, with area larger than (C). TIA, transient ischemic attack; DW1, diffusion-weighted imaging; MRA, magnetic resonance angiography; ASL, arterial spin labeling.

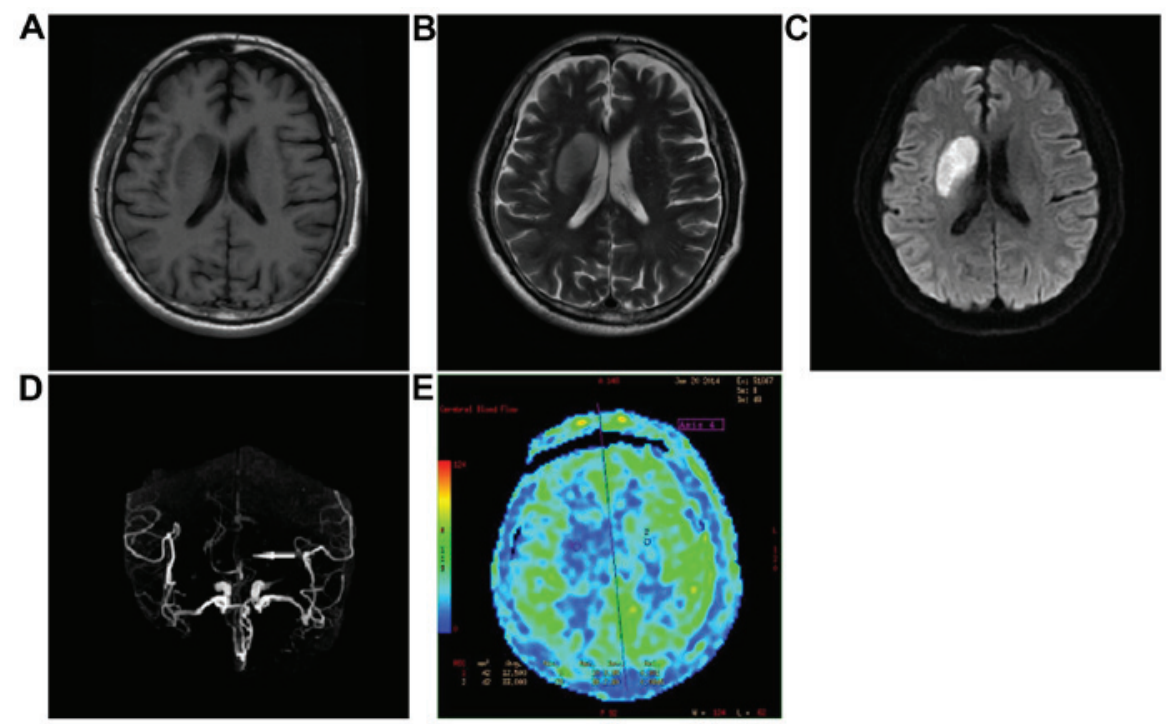

Figure 2. Cerebral apoplexy scan (A) T1. (B) T2, which shows a large area of infarcted focus at right basal ganglia region. (C) DW1 showing high density shadow of the same position. (D) MRA showing absence and interruption of left posterior cerebral artery. (E) ASL, rCBF=0.4, blue color is the hypoperfusion area and the area is larger than that in (C). DW1, diffusion-weighted imaging; MRA, magnetic resonance angiography; ASL, arterial spin labeling.

6 cases were at cerebellum and 3 cases were at brainstem. A total of 13 cases of abnormal signal in conventional MRI were identified through ASL technology. Diameter stenosis beyond 30\% in MRA were tested in ASL. A positive rate in ASL was significantly higher than that of conventional MRI $\left(\chi^{2}=29.078, \mathrm{P}<0.001\right)$ and the hypoperfusion area was greatly increased $(\mathrm{t}=32.526, \mathrm{P}<0.001)$. The $\mathrm{rCBF}$ value was positively correlated with the degree of diameter stenosis shown in MRA ( $\mathrm{r}=0.524, \mathrm{P}=0.012)$. Additionally, the positive rate of ASL was positively correlated with the attack times of TIA ( $\mathrm{r}=0.352, \mathrm{P}=0.027$ ) (Fig. 1).

Analysis on the development in cerebral apoplexy group. There were 39 cases of abnormal signal tested in conventional MRI (manifesting as T1 and T2 low signal) and the positive rate was $65.0 \%$, with a hypoperfusion area of DWI was $1.6-4.5 \mathrm{~mm}^{2}$ and an average was $3.2 \pm 1.3 \mathrm{~mm}^{2}$. The diameter stenosis of intracranial arterial lumen tested in MRA was 30-75\% and the average was $56.5 \pm 14.8 \%$. In ASL, 52 cases had a hypoperfusion area $(86.7 \%)$, rCBF $0.4-1.0$ and an average of $0.6 \pm 0.2$. The rate of abnormal perfusion signals was $91.7 \%$ (55/60), the hypoperfusion area was $1.8-8.5 \mathrm{~mm}^{2}$ and the average was $5.7 \pm 1.6 \mathrm{~mm}^{2}$. For the location of the hypoperfusion area, 26 cases were at basal ganglia, 14 cases at cerebrum, 7 cases at cerebellum and 5 cases at brainstem. A total of 39 cases of abnormal signal in the conventional MRI were identified through ASL technology. A positive rate in ASL was significantly higher than that of the conventional MRI $\left(\chi^{2}=7.685, \mathrm{P}=0.006\right)$ and the hypoperfusion 
area was greatly increased $(\mathrm{t}=9.425, \mathrm{P}<0.001)$. The $\mathrm{rCBF}$ value was positively correlated with the degree of diameter stenosis ( $\mathrm{r}=0.635, \mathrm{P}=0.009$ ) (Fig. 2).

\section{Discussion}

ASL is a non-invasive, safe and simple technology that tests blood perfusion of brain tissue. Decrease or abnormality of brain blood perfusion was the pathological basis of ischemic cerebrovascular disease and the main diagnostic basis. There were corresponding clinical symptoms when whole-brain CBF was $<50 \%$, in other words $23 \mathrm{ml} / \mathrm{min} \cdot 100 \mathrm{~g}(9)$. ASL technology was used to test the decrease or absence of perfusion in the early period and calculate $\mathrm{rCBF}$, which provided a significant reference value for the quantitative reaction of hemodynamic changes of cerebrovascular diseases as well as clinical diagnosis and treatment (10). Based on different inversion labeling methods for arterial blood, ASL technology was divided into continuous ASL (CASL) and pulsing ASL (PASL). CASL has a higher SNR and narrow labeling plane, its sensitive area of radiofrequency coil need not be long and consequently, it is considered more suitable for a lower anatomical layer (5). However, CASL has a higher radiofrequency energy deposition and its tissue was greatly influenced by the magnetization transfer effect (11). Nevertheless, PASL was able to eliminate the magnetization vector from static structure at the notch pulse and control pulse, which may minimize defective artifacts of profiled outline. In addtion, the inversion labeling rate of PASL is greatly improving (12). With the development of MR software and hardware and increasing investigations of ASL technology, the ASL sequence is also improving continuously, such as FAIREST technology performing the third imaging on imaging layer after saturation based on core sequence (FAIR sequence), which is able to effectively separate blood oxygen level-dependent (BOLD) weight and perform quantitative CBF measurement (13). BOLD and T1-weighted imaging can subsequently be obtained to reflect blood perfusion and metabolic status of brain tissue more accurately (13).

Calamante et al applied CASL technology to examine transient middle cerebral artery occlusion in a rat model (14). The results of that study showed that, ASL can be used to reveal low blood perfusion of cerebral ischemia for animals and monitor the time course of ischemia reperfusion and treatment effects of embolism (14). Chalela et al (15) have previously reported a CASL study with 15 cases of acute ischemic apoplexy patients. The findings of that study showed that CASL is able to identify insufficient perfusion of acute cerebral ischemia and is correlated with clinical symptoms (15). In addition, Bokkers et al (16) used ASL technology to scan 23 cases of carotid artery stenosis (including cases with and without ischemic symptoms) and 20 healthy cases and observed the perfusion of brain tissue prior to and after acetazolamide. The results of those authors showed that the perfusion area of brain tissue for carotid artery stenosis with symptoms was smaller than those with no symptoms (16). After intravenous injection of acetazolamide, cerebral blood perfusion increased, but the increase in scope for carotid artery stenosis with symptoms were lower than that of cases with symptoms, but both of them were lower than those of healthy individuals. The results of that study indicated that ASL was able to test blood perfusion of brain tissue, providing CBF information for cerebral ischemia patients, which was useful in conventional MRI testing.

The 13 cases of abnormal signal in conventional MRI (positive rate 22.4\%) were also identified through ASL technology (positive rate 63.8\%). Diameter stenosis beyond $30 \%$ in MRA was also tested in ASL. The positive rate in ASL was significantly higher than that of conventional MRI and hypoperfusion area was greatly increased. The $\mathrm{rCBF}$ value was positively correlated with the degree of diameter stenosis shown in MRA and the positive rate of ASL was positively correlated with the attack times of TIA. A total of 39 abnormal signal cases of cerebral apoplexy in conventional MRI (positive rate 65.0\%) were identified through ASL technology (positive rate $86.7 \%$ ). A positive rate in ASL was significantly higher than that of the conventional MRI and hypoperfusion area was greatly increased. The rCBF value was positively correlated with the degree of diameter stenosis. In conclusion, 3.0T ASL was equally important in the early diagnosis of TIA and mild, moderate intracranial arterial stenosis of cerebral apoplexy.

\section{References}

1. Bu X, Li C, Zhang Y, Xu T, Wang D, Sun Y, Peng H, Xu T, Chen CS, Bazzano LA, Chen J and He J; CATIS Investigators. Early Blood Pressure Reduction in Acute Ischemic Stroke with Various Severities: A subgroup analysis of the CATIS trial. Cerebrovasc Dis 42: 186-195, 2016.

2. Psychogios K, Stathopoulos P, Takis K, Vemmou A, Manios E, Spegos K and Vemmos K: The Pathophysiological Mechanism Is an independent predictor of long-term outcome in stroke patients with large vessel atherosclerosis. J Stroke Cerebrovasc Dis 24: 2580-2587, 2015

3. Kivikko M, Kuoppamäki M, Soinne L, Sundberg S, Pohjanjousi P, Ellmen J and Roine RO: Oral levosimendan increases cerebral blood flow velocities in patients with a history of stroke or transient ischemic attack: A pilot safety study. Curr Ther Res Clin Exp 77: 46-51, 2015.

4. Snow NJ, Peters S, Borich MR, Shirzad N, Auriat AM, Hayward KS and Boyd LA: A reliability assessment of constrained spherical deconvolution-based diffusion-weighted magnetic resonance imaging in individuals with chronic stroke. J Neurosci Methods 257: 109-120, 2016.

5. Zhang SX, Yao YH, Zhang S, Zhu WJ, Tang XY, Qin YY, Zhao LY, Liu CX and Zhu WZ: Comparative study of DSC-PWI and 3D-ASL in ischemic stroke patients. J Huazhong Univ Sci Technolog Med Sci 35: 923-927, 2015.

6. Eugène F, Gauvrit JY, Ferré JC, Gentric JC, Besseghir A, Ronzière $\mathrm{T}$ and Raoult $\mathrm{H}$ : One-year $\mathrm{MR}$ angiographic and clinical follow-up after intracranial mechanical thrombectomy using a stent retriever device. AJNR Am J Neuroradiol 36: 126-132, 2015.

7. Faraco CC, Strother MK, Dethrage LM, Jordan L, Singer R, Clemmons PF and Donahue MJ: Dual echo vessel-encoded ASL for simultaneous BOLD and CBF reactivity assessment in patients with ischemic cerebrovascular disease. Magn Reson Med 73: 1579-1592, 2015.

8. Nael K, Meshksar A, Liebeskind DS, Wang DJ, Ellingson BM, Salamon N and Villablanca JP; UCLA Stroke investigators: Periprocedural arterial spin labeling and dynamic susceptibility contrast perfusion in detection of cerebral blood flow in patients with acute ischemic syndrome. Stroke 44: 664-670, 2013.

9. Wang DJ, Alger JR, Qiao JX, Gunther M, Pope WB, Saver JL, Salamon N and Liebeskind DS; UCLA Stroke Investigators: Multi-delay multi-parametric arterial spin-labeled perfusion MRI in acute ischemic stroke - Comparison with dynamic susceptibility contrast enhanced perfusion imaging. Neuroimage Clin 3: 1-7, 2013. 
10. Kaichi Y, Okada G, Takamura M, Toki S, Akiyama Y, Higaki T, Matsubara Y, Okamoto Y, Yamawaki S and Awai K: Changes in the regional cerebral blood flow detected by arterial spin labelingafter 6-week escitalopram treatment for major depressive disorder. J Affect Disord 194: 135-143, 2016.

11. Garraux G, Hallett $M$ and Talagala SL: CASL fMRI of subcortico-cortical perfusion changes during memory-guided finger sequences. Neuroimage 25: 122-132, 2005.

12. Bibic A, Knutsson L, Schmidt A, Henningsson E, Månsson S, Abul-Kasim K, Ảkeson J, Gunther M, Ståhlberg F and Wirestam R: Measurement of vascular water transport in human subjects using time-resolved pulsed arterial spin labelling. NMR Biomed 28: 1059-1068, 2015.

13. Guo L, Zhang Q, Ding L, Liu K, Ding K, Jiang C, Liu C, Li K and Cui L: Pseudo-continuous arterial spin labeling quantifies cerebral blood flow in patients with acute ischemic stroke and chronic lacunar stroke. Clin Neurol Neurosurg 125: 229-236, 2014.
14. Calamante F, Thomas DL, Pell GS, Wiersma J and Turner R: Measuring cerebral blood flow using magnetic resonance imaging techniques. J Cereb Blood Flow Metab 19: 701-735, 1999.

15. Chalela JA, Alsop DC, Gonzalez-Atavales JB, Maldjian JA, Kasner SE and Detre JA: Magnetic resonance perfusion imaging in acute ischemic stroke using continuous arterial spin labeling. Stroke 31: 680-687, 2000

16. Bokkers RPH, van Osch MJP, van der Worp HB, de Borst GJ, Mali WP and Hendrikse J: Symptomatic carotid artery stenosis: Impairment of cerebral autoregulation measured at the brain tissue level with arterial spin-labeling MR imaging. Radiology 256: 201-208, 2010. 